\title{
Radiation-induced sacral insufficiency fracture in endometrial cancer patient after adjuvant radiotherapy: a case report
}

\author{
Yen-Ting Liu ${ }^{1}$, Sung-Hsin Kuo ${ }^{1}$, Ting-Chen Chang $^{2}$, Chao-Yuan Huang ${ }^{1,3}$ \\ ${ }^{1}$ Division of Radiation Oncology, Department of Oncology, National Taiwan University Hospital and National Taiwan University College of \\ Medicine, Taipei, Taiwan; ${ }^{2}$ Department of Obstetrics and Gynecology, National Taiwan University Hospital, Taipei, Taiwan; ${ }^{3}$ Department of \\ Radiological Technology, Yuanpei University, Hsinchu, Taiwan \\ Correspondence to: Chao-Yuan Huang, MD. Department of Oncology, National Taiwan University Hospital and National Taiwan University College \\ of Medicine., No. 1, Changde St., Zhongzheng Dist., Taipei City 10048, Taiwan. Email: cyhuang999@ntu.edu.tw.
}

\begin{abstract}
Sacral insufficiency fracture (SIF) is a common cause of back pain in the elderly. However, SIF is still under-diagnosis. We presented a patient with endometrial cancer who complained of low back pain after adjuvant radiotherapy. Finally, SIF was detected by magnetic resonance imaging (MRI). This 66-year-old woman was diagnosed with endometrioid adenocarcinoma, stage Ia, grade 3 . She received staging surgery (total abdominal hysterectomy, bilateral salpingo-oophorectomy, bilateral pelvic lymph nodes dissection, omental biopsy and peritoneal washing cytology) followed by adjuvant radiotherapy with the dose of 50.4 Gy in 28 fractions to pelvic lymphatics and vaginal stump (from November 2014 to December 2014). After completion of radiotherapy for 6 months, she complained of diffuse low back pain, but denied any trauma history. The results of physical examination for pelvic and rectal area were normal. The abdomenpelvis computer tomography revealed no significant finding. The whole body bone scans disclosed the suspect bone metastases at left sacroiliac joint (SI joint). Considering the osteoporosis-associated fracture is common present in elderly women, we perform the MRI of SI joint to differentiate whether these lesions are benign process or malignancy. The result of MRI confirmed the diagnosis of radiation-associated SIF which is characterized by the signs of marrow edema (more obvious by STIR, Short T1 Inversion Recovery, MRI images). She received supportive care and had back pain subsided during regular follow-up. With the accurate diagnosis of SIF but not bony metastasis, this patient had subsequent favorable clinical course and outcome with resolution of symptoms by conservative treatment.
\end{abstract}

Keywords: Radiotherapy; sacral insufficiency fracture (SIF); endometrial cancer; case report

Received: 14 November 2017; Accepted: 23 February 2018; Published: 30 June 2020.

doi: $10.21037 /$ tro-17-tastro-16

View this article at: http://dx.doi.org/10.21037/tro-17-tastro-16

\section{Introduction}

Sacral insufficiency fracture (SIF) occurs when normal stress placed on weakened bone, which presents with low back or pelvic pain. SIF was first described in 1982 by Lourie (1). Elderly women with osteoporosis are thought to be the highest group of SIF (2). Most of patients are over 55 years old, and the majority of them are between 70 and 75 years old. Other risk factors include previous pelvic radiotherapy (RT) history, steroid-induced osteopenia, hyperparathyroidism, and multiple myeloma (2-4). RT may increase fracture risks by the effect of bone damage. The prospective randomized trials
(Stockholm I and Stockholm II) revealed that rectal cancer patients received $\mathrm{RT}$ increased the incidence of femoral neck and pelvic fractures $(\mathrm{P}=0.03)(5-7)$. Uezono et al. reported that the 2-year cumulative incidence of pelvic insufficiency fracture was $32 \%$ for cervical cancer patients after definitive RT (8). For the cervical and endometrial cancer patients after postoperative pelvic RT, those who with osteoporosis, the pelvic insufficiency fracture rate at 5 -year was $15.6 \%$ compared with $2.9 \%$ for those without osteoporosis $(\mathrm{P}=0.01)(9)$.

Pain is the common presentation of SIF, especially for diffuse low back pain, which may radiates to the hip or 


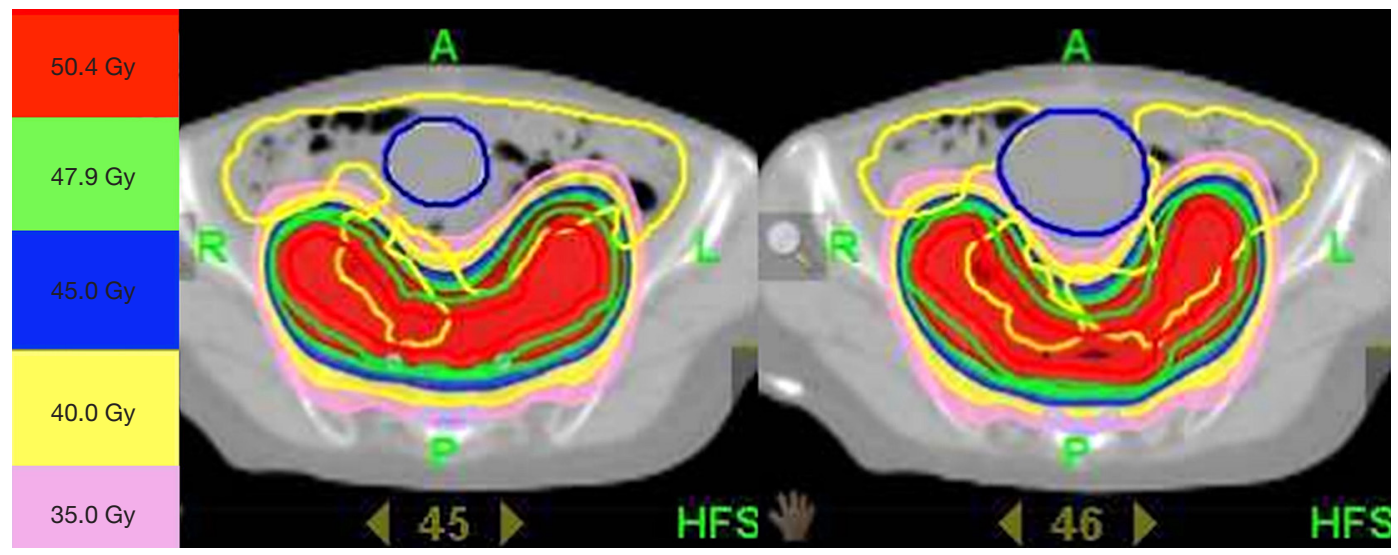

Figure 1 The isodose curve is shown above using tomotherapy.

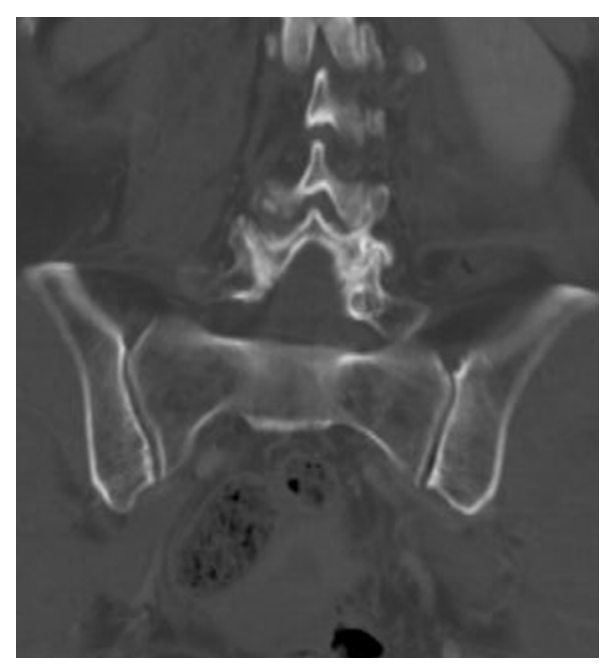

Figure 2 No obvious bone abnormality on coronal view of pelvis CT in 2014/7.

buttock with intractable or acute pattern, and it is often confused with other diagnosis in the elderly $(3,4,10,11)$. Low back pain in the elderly might confuse clinicians with radiculopathy, bone metastases, or possible occult cancer, so the differential diagnosis is important. Now with multiple established image findings (including CT scan, bone scintigraphy and MR imaging) help us to make assurance of SIF. We presented a patient with endometrial cancer who complained of low back pain after adjuvant RT. Finally, SIF was detected by magnetic resonance imaging (MRI).

We present the following case in accordance with the CARE reporting checklist (available at http://dx.doi. org/10.21037/tro-17-tastro-16).

\section{Case presentation}

This 67-year-old woman was diagnosed with endometrioid adenocarcinoma, pT1aN0M0, stage Ia, grade 3 in 2014. She received staging surgery (total abdominal hysterectomy, bilateral salpingo-oophorectomy, bilateral pelvic lymph nodes dissection, omental biopsy and peritoneal washing cytology) followed by adjuvant RT. The setting was helical therapy (Tomotherapy) technique with $6 \mathrm{MV}$ photon. RT fields based on RTOG Gynecologic IMRT Consortium (12) included vaginal cuff, parametrial tissues, common iliac, external, and internal iliac nodal regions (Figure 1). The prescribed dose was 50.4 Gy in 28 fractions from November 2014 to December 2014. Then, she was under regular follow-up at radiation oncology and gynecology department. After completion of RT for 6 months, she complained of diffuse low back pain without neurological symptom, but denied any trauma history. Bone metastasis was impressed. However, reviewing her CT of abdomen and pelvis on July 30th, 2014 before treatment, it showed no fracture lines, cortical destruction, neither obvious sclerosis of SI joints (Figure 2). The physical examination revealed non-specific knocking tenderness over low back which cannot be precisely localized. In addition, the pelvic and rectal examination were all normal.

The gynecology oncologist arranged the X-ray examination (plain films, anterior-posterior/lateral) of thoracic-spine and lumbar-spine, and the bone mineral density test to rule out osteoporosis. The result of the plain films showed spondylolisthesis, and T-score of the bone mineral density test was -2.6 , indicating osteoporosis $(<-2.5)$. Due to her cancer history, the abdomen-pelvis 

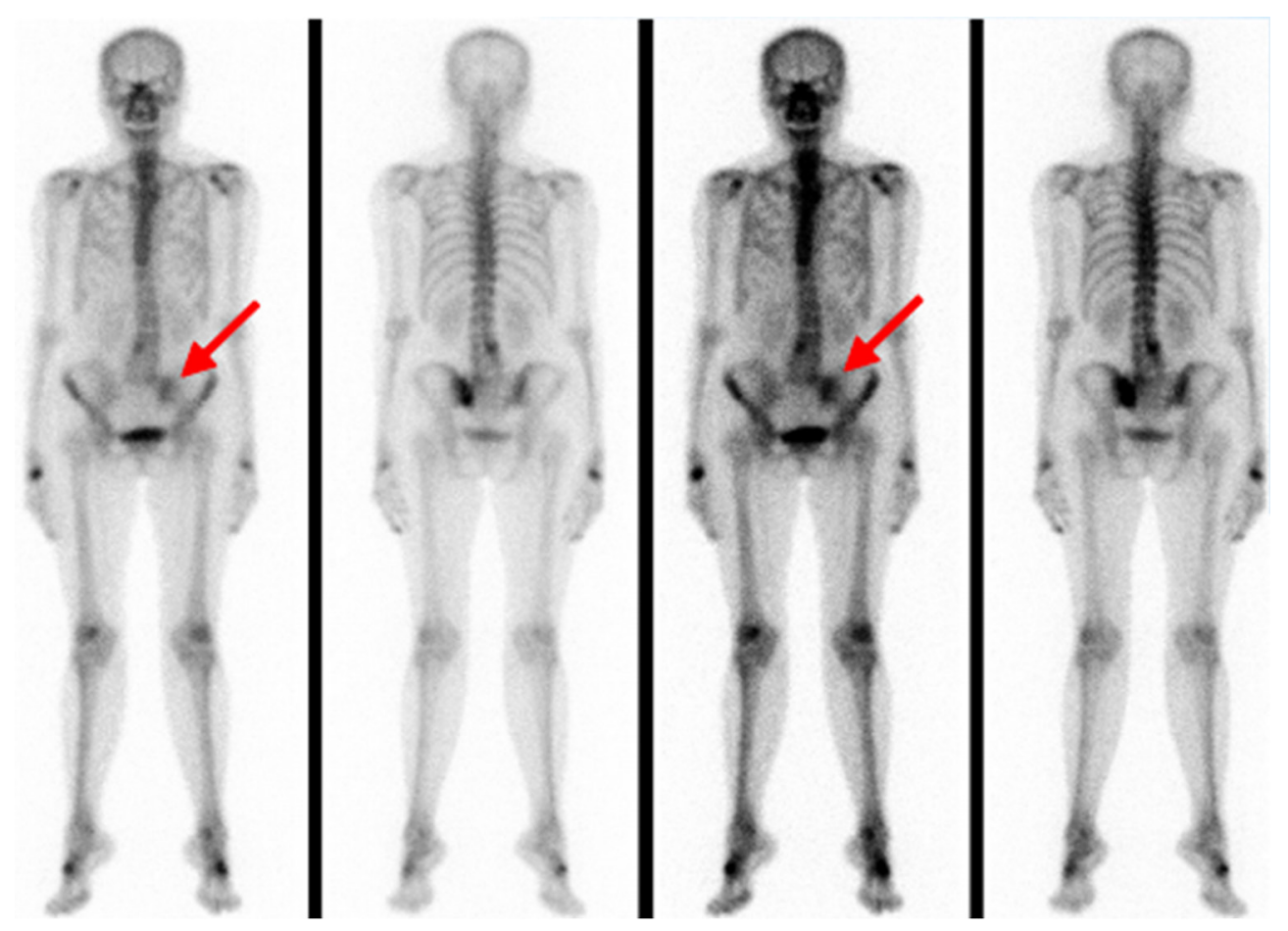

Figure 3 The focal hot area at left sacroiliac joint is shown on whole body bone scintigraphy (red arrows).

computer tomography and bone scintigraphy was arranged for survey. The computer tomography revealed no significant finding, but the whole body bone scan disclosed the suspect bone metastases at left sacroiliac joint (SI joint) (Figure 3). Considering the osteoporosis-associated fracture is a common present in elderly women, we perform the MRI of SI joint to differentiate whether these lesions are benign process or malignancies. The results of MRI confirmed the diagnosis of radiation-associated SIF. The SIF is characterized by the signs of marrow edema which noted by Short T1 Inversion Recovery (STIR) MR images (Figure 4). The conservative treatment with bed rest and simple opioid analgesia was given. After supportive care for 4 months, her back pain subsided during regular follow-up, and she felt well. In addition, the CT in March, 2016 after completion of RT for one year demonstrated some bone sclerosis and equivocal fracture lines, seemed bone union (Figure 5).

\section{Discussion}

Insufficiency fractures are stress fractures due to normal stress applied to abnormally weakened bone that has lost its elastic resistance (13). The most common site is the sacrum ala, known as a SIF (14).

The common cause of SIF is post-menopausal osteoporosis. However, RT become an essential treatment of gynecologic cancers, and prior pelvic RT is also reported as a risk factor, either definitive or adjuvant treatment $(4,8,9,15)$. The prevalence of SIF is $21-34 \%$ in the population with prior pelvic radiation $(4,15,16)$.

Shih et al. demonstrated that 222 patients of cervical or endometrial cancer treated with postoperative pelvic RT, who has osteoporosis or prior hormone-replacement therapy developed higher risk of pelvic insufficiency fractures $(15.6 \% v s$. $2.9 \%, \mathrm{P}=0.01$ and $14.8 \%$ vs. $4.1 \%, \mathrm{P}=0.009$, respectively) (9). Even with modern technique of intensity-modulated radiation therapy (IMRT), there is no difference in the rate of pelvic insufficiency fractures comparing with conventional RT (9). Retrospective study of SEER database with 6,428 women older than 65 years revealed that 5 -year cumulative pelvic fracture rate significantly increased in who received pelvic irradiation: $8.2 \%$ vs. $5.9 \%$ (hazard ratio, 1.66 ; $95 \%$ confidence interval, 1.06-2.59) (17).

Injury and occlusion of the microvasculature of bone and stasis of osteoclasts are thought to be major mechanisms of radiation-induced insufficiency fracture. It is known as "radiation osteitis" which includes a radiation-induced 


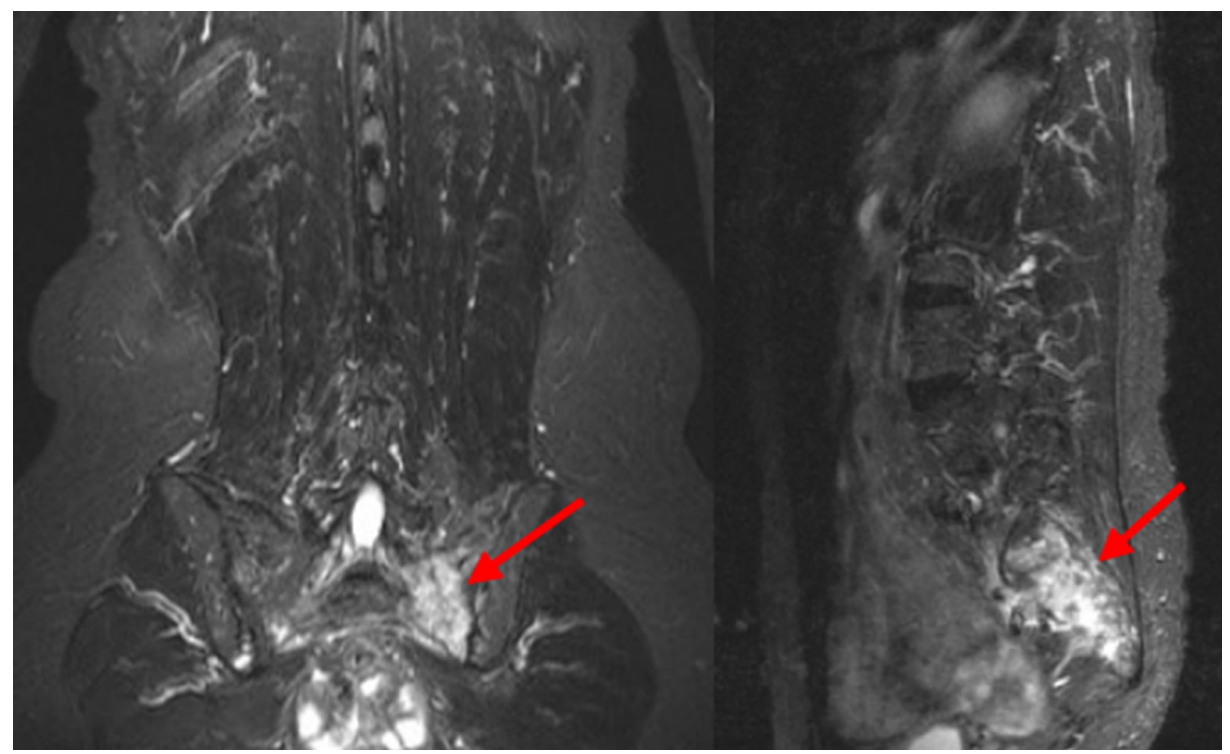

Figure 4 The signs of marrow edema at left sacroiliac joint are obvious by STIR (Short T1 Inversion Recovery) MRI (red arrows).

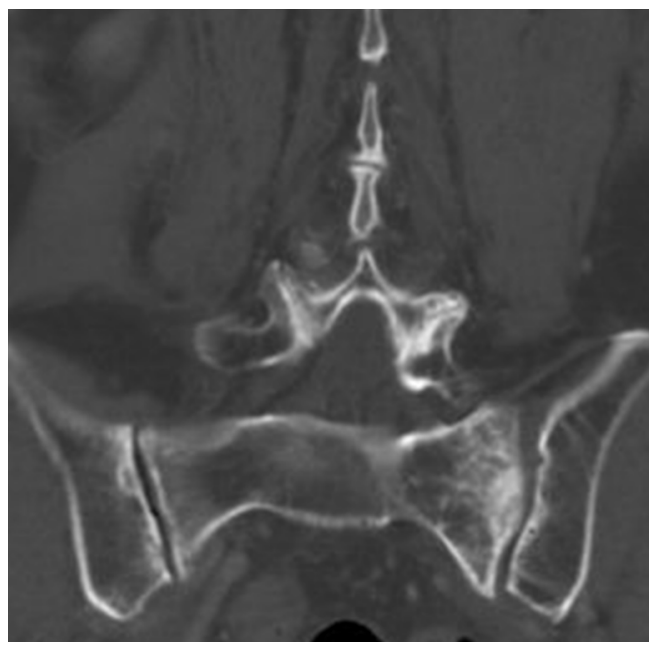

Figure 5 Bone union of left SI joint on coronal view of pelvis CT in March, 2016.

inflammatory response in blood vessels, nerve tissue, and bone cells that may lead to fracture of trabecular and cortical bone (18).

SIF may be presented with fracture lines and bone sclerosis by plain films or CT imaging. Plain films with anterior-posterior \& lateral are not good detectable tools due to the obscurity of bowel gas or invalid fracture lines. The computed tomography of pelvis can well demonstrate fracture lines and sclerosis, and differentiate from cortical destruction and/or a soft-tissue mass in cases of tumor involvement. However, the diagnostic sensitivity of SIF by computed tomography is around $60 \%$ to $75 \%$ because early signs of SIF on CT is not obvious $(14,19)$. The bone scintigraphy has good sensitivity about $96 \%$ for diagnosing SIF by presenting with $\mathrm{H}$-pattern (20-40\% of patients) $(16,20)$. Nevertheless, it can be misdiagnosed as isolated bony metastases, especially in the population with previous cancer history. The MRI is useful for early detection of SIF. It can provide the same sensitivity as bone scintigraphy and can differentiate SIF from bony metastases if there is tumor mass identified $(14,19,21)$. The marrow edema is early sign of SIF seen on the MR imaging, especially in STIR MR images.

The treatments for SIF are analgesic medication and bed rest. The conservative management can help most patients return to their normal life in a period of time. Only some patients with instable fracture or intractable pain may need surgery. The sacroplasty treatment reported good symptoms relief in SIF patients (14).

Although one case is in the present study, SIF is still under-diagnosis, especially among cancer patients due to malignant history. Besides, a lot of studies demonstrated the same findings, including long-term follow-up of Stockholm I and Stockholm II trials (6).

In this case report, we demonstrated an endometrial cancer patient with osteoporosis who received adjuvant RT with low back pain after 6 months of pelvic RT. Whole body 
bone scans disclosed the suspect bone metastases at SI joint. However, with the accurate diagnosis of SIF by MRI, this patient had subsequent favorable clinical course and outcome with resolution of symptoms by conservative treatment.

\section{Acknowledgments}

Funding: None.

\section{Footnote}

Reporting Checklist: The authors have completed the CARE reporting checklist. Available at http://dx.doi.org/10.21037/ tro-17-tastro- 16.

Conflicts of Interest: All authors have completed the ICMJE uniform disclosure form (available at http://dx.doi. org/10.21037/tro-17-tastro-16). SHK serves as an Associate Editors-in-Chief of Therapeutic Radiology and Oncology from Apr 2020 to Mar 2022. CYH serves as the unpaid editorial board member of Therapeutic Radiology and Oncology from Apr 2020 to Mar 2022. The other authors have no conflicts of interest to declare.

Ethical Statement: The authors are accountable for all aspects of the work in ensuring that questions related to the accuracy or integrity of any part of the work are appropriately investigated and resolved. All procedures performed in studies involving human participants were in accordance with the ethical standards of the institutional and/or national research committee(s) and with the Helsinki Declaration (as revised in 2013). Written informed consent was obtained from the patient for publication of this manuscript and any accompanying images.

Open Access Statement: This is an Open Access article distributed in accordance with the Creative Commons Attribution-NonCommercial-NoDerivs 4.0 International License (CC BY-NC-ND 4.0), which permits the noncommercial replication and distribution of the article with the strict proviso that no changes or edits are made and the original work is properly cited (including links to both the formal publication through the relevant DOI and the license). See: https://creativecommons.org/licenses/by-nc-nd/4.0/.

\section{References}

1. Lourie H. Spontaneous osteoporotic fracture of the sacrum. JAMA 1982;248:715-7.

2. Isdale AH. Sacral insufficiency fractures: an unsuspected cause of low back pain. Rheumatology (Oxford) 1999;38:90.

3. De Smet AA, Neff JR. Pubic and sacral insufficiency fractures: Clinical course and radiologic findings. AJR Am J Roentgenol 1985;145:601-6.

4. Gotis-Graham I, McGuigan L, Diamond T, et al. Sacral insufficiency fractures in the elderly. J Bone Joint Surg Br 1994;76:882-6.

5. Cedermark B, Johansson H, Rutqvist LE, et al. The Stockholm I trial of preoperative short term radiotherapy in operable rectal carcinoma: a prospective randomized trial. Stockholm Colorectal Cancer Study Group. Cancer 1995;75:2269-75.

6. Holm T, Singnomklao T, Rutqvist LE, et al. Adjuvant preoperative radiotherapy in patients with rectal carcinoma: adverse effects during long term follow-up of two randomized trials. Cancer 1996;78:968-76.

7. Stockholm Colorectal Cancer Study Group. Randomized study on preoperative radiotherapy in rectal carcinoma. Ann Surg Oncol 1996;3:423-30.

8. Uezono H, Tsujino K, Moriki K, et al. Pelvic insufficiency fracture after definitive radiotherapy for uterine cervical cancer: Retrospective analysis of risk factors. J Radiat Res 2013;54:1102-9.

9. Shih KK, Folkert MR, Kollmeier MA, et al. Pelvic insufficiency fractures in patients with cervical and endometrial cancer treated with postoperative pelvic radiation. Gynecol Oncol 2013;128:540-3.

10. Peh WC, Khong PL, Ho WY, et al. Sacral insufficiency fractures. spectrum of radiological features. Clin Imaging 1995;19:92-101.

11. Rawlings CE 3rd, Wilkins RH, Martinez S, et al. Osteoporotic sacral fractures: a clinical study. Neurosurgery 1988;22:72-6.

12. Lim K, Small W Jr, Portelance L, et al. Consensus guidelines for delineation of clinical target volume for intensity-modulated pelvic radiotherapy for the definitive treatment of cervix cancer. Int $\mathbf{J}$ Radiat Oncol Biol Phys 2011;79:348-55.

13. Pentecost RL, Murray RA, Brindley HH. Fatigue, insufficiency, and pathologic fractures. JAMA 1964;187:1001-4.

14. Lyders EM, Whitlow CT, Baker MD, et al. Imaging and treatment of sacral Insufficiency fractures. AJNR Am J Neuroradiol 2010;31:201-10.

15. Oh D, Huh SJ. Insufficiency fracture after radiation 
therapy. Radiat Oncol J 2014;32:213-20.

16. Abe H, Nakamura M, Takahashi S, et al. Radiationinduced insufficiency fractures of the pelvis: evaluation with 99m Tc-methylene diphosphonate scintigraphy. AJR Am J Roentgenol 1992;158:599-602.

17. Baxter NN, Habermann EB, Tepper JE, et al. Risk of pelvic fractures in older women following pelvic irradiation. JAMA 2005;294:2587-93.

18. Hopewell JW. Radiation-therapy effects on bone density. Med Pediatr Oncol 2003;41:208-11.

doi: $10.21037 /$ tro-17-tastro-16

Cite this article as: Liu YT, Kuo SH, Chang TC, Huang CY. Radiation-induced sacral insufficiency fracture in endometrial cancer patient after adjuvant radiotherapy: a case report. Ther Radiol Oncol 2020;4:10.
19. Cabarrus MC, Ambekar A, Lu Y, et al. MRI and CT of Insufficiency fractures of the pelvis and the proximal Femur. AJR Am J Roentgenol 2008;191:995-1001.

20. Fujii M, Abe K, Hayashi K, et al. Honda sign and variants in patients suspected of having a sacral Insufficiency fracture. Clin Nucl Med 2005;30:165-9.

21. Blomlie V, Rofstad EK, Talle K, et al. Incidence of radiation-induced insufficiency fractures of the female pelvis: evaluation with MR imaging. AJR Am J Roentgenol 1996;167:1205-10. 